\title{
Prevención de los comportamientos sexuales de riesgo en los adolescentes: SIDA, otras enfermedades de transmisión sexual y embarazos no deseados
}

\author{
Montserrat Planes \\ Universitat Autónoma de Barcelona
}

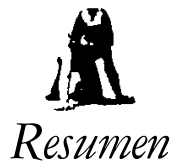

En la actualidad, los adolescentes son uno de los grupos que por sus características conductuales, cognitivas y sociales, se encuentran en mayor riesgo frente al posible contagio con el VIH. Esta amenaza para la saludy el bienestar de los adolescentes, se ba venido a sumar a otros problemas ya existentes en este colectivo: los embarazos no deseados y las enfermedades de transmisión sexual (ETS), que también se derivan de la no utilización de precauciones en las relaciones sexuales.

En este trabajo se exponen diversos factores biológicos, psicológicos (conductuales y cognitivos) y sociales que pueden facilitar, dificultar o impedir los comportamientos sexuales de prevención de los adolescentes, y se revisan y valoran algunas de las intervenciones preventivas realizadas. A partir de esta revisión se argumenta sobre la conveniencia de unificar los programas para la prevención simultánea de los tres trastornos, maximizando de esta forma los recursos disponibles.

Palabrasclave: Adolescentes, SIDA, comportamientossexuales de riesgo.

\section{Preventing sexual risk behaviours in adolescents: AIDS, other sexually transmitted disease, and unwanted pregnancies}

\section{Abstract}

At present, adolescents are a group which due to their behavioural, cognitive, and social characteristics, run the greatest risk of possible contagion. This threat to the health and well-being of adolescents is added to other problems already facing them, such as unwanted pregnacies and sexually transmitted diseases (STD), which are due to the non-use of preventive methods in sexual relations. The paper deals with biological, psychological (behavioural and cognitive), and social factors that may facilitate, interfere with or impede preventive sexual behaviours in adolescents. Some preventive intervention programmes are also reviewed and assessed. The discussion supports the advisability of unifying programmes aimed at the simultaneous prevention of all three risk areas to optimize available resources.

Keywords: Adolescents, AIDS, sexual-risk behaviour.

Agradecimientos: Este trabajo ha sido realizado gracias a la ayuda PB 89-0312 de la Dirección de Investigación Científica y Técnica del Ministerio de Educación y Ciencia.

Correspondencia con la autora: Departamento de Psicología de l'Educació. Universitat Autónoma de Barcelona. Estudi General de Girona. Plaça Sant Domenech, 9. 17001 Girona.

Original recibido: Julio de 1992. Revisión recibida: Enero de 1994. Aceptado: Marzo de 1994. 


\section{INTRODUCCIÓN}

La salud de los adolescentes y, en particular, los trastornos inmediatos o demorados que padecen a causa de la realización de comportamientos de riesgo (fumar, beber alcohol, conducir vehículos de forma imprudente, mantener relaciones sexuales sin protección, etc.), se ha convertido, en estos últimos años, en uno de los temas de interés preferente para los profesionales de la salud y de la educación (Basch, 1989; Cáceres et al., 1992; Mendoza, 1984).

Uno de los factores que, probablemente, ha contribuido a que se incremente la preocupación por la salud de los adolescentes, ha sido la constatación a lo largo de la década de los 80 — en el inicio de la cual se conocieron los primeros casos de Sida provocados por el virus de inmunodeficiencia humana (VIH) — de que esta enfermedad mortal se iba extendiendo y afectando a cualquier colectivo humano, poniendo de manifiesto que su padecimiento no quedaba limitado a los llamados «grupos de riesgo» (Brooks-Gunn et al., 1988; Weisse et al., 1990).

En la actualidad, aunque todavía no existen fármacos eficaces para prevenir o curar el Sida, ya se conocen con precisión, tanto el virus causante de la enfermedad, como las principales vías de contagio con el mismo: sangre/sangre, sangre/semen/secreciones vaginales y materno/ filial. Por consiguiente, dado que el virus se transmite a través de un número limitado de comportamientos, la infección se puede evitar eficazmente realizando conductas preventivas, en especial, las relacionadas con la utilización de material de inyectar estéril y con el uso de preservativo durante las relaciones sexuales (Cf.: Bayés, 1992).

Teniendo en cuenta que la adolescencia es una edad de experimentación, durante la cual los sujetos suelen iniciar sus relaciones sexuales y también los contactos con la droga, estos comportamientos les ponen doblemente en riesgo de infectarse con el VIH (Bowler et al., 1992; Weisse et al., 1990).

En este trabajo nos vamos a ocupar básicamente de los comportamientos sexuales de los adolescentes, aunque también examinaremos las influencias negativas del uso de drogas sobre los comportamientos sexuales de prevención.

Varios motivos nos llevan a prestar mayor atención a los comportamientos sexuales. Aparte de que afectan a un mayor número de adolescentes y de que ambos sexos están implicados en la misma medida, la posible infección con el virus del Sida a través de las relaciones sexuales ha venido a sumarse a otras consecuencias negativas producidas por la falta de prevención: las enfermedades de transmisión sexual clásicas (ETS) y los embarazos no deseados (Boyer y Kegeles, 1991; Cates y Bowen, 1989).

Teniendo presentes estas consideraciones centraremos los objetivos de nuestro trabajo fundamentalmente en :

1) Poner de manifiesto la importancia de las consecuencias negativas derivadas de los comportamientos sexuales de riesgo de los adolescentes respecto a la infección con el VIH, otras ETS y embarazos no deseados, mediante la revisión de los datos epidemiológicos.

2) Analizar una serie de factores bio-psico-sociales que han mostrado su influencia, en diferentes investigaciones realizadas con adolescentes, sobre los comportamientos sexuales de prevención y de riesgo.

\section{EPIDEMIOLOGIA}

Según los Centers of Disease Control (CDC), la adolescencia abarca desde los 13 a los 19 años, mientras que la American Academy of Pediatrics and Society 


\section{7}

for Adolescent prolonga este periodo hasta los 21 años (Cf.: Bowler et al., 1992). En este trabajo nos ocuparemos preferentemente de los más jóvenes.

\section{Sida}

En primer lugar, hay que precisar que sólo existen datos epidemiológicos sobre la incidencia y prevalencia de la enfermedad manifiesta, debido a la obligatoriedad de declarar a las autoridades sanitarias todos los casos diagnosticados, aunque preservando el anonimato de los afectados. A partir de estos datos y de los estudios serológicos con grupos limitados se realizan estimaciones sobre el número total de personas que pueden estar infectadas. Según Blake (1991), se cree que hay alrededor de 10 infectados $(\mathrm{VIH}+)$ por cada caso de Sida diagnosticado.

En España, los datos publicados por la OMS (1993a) muestran que, hasta el 31 de marzo de 1993, la prevalencia del Sida entre los adolescentes de 13 a 19 años es: 214 de un total de 18.347 enfermos de todas las edades, lo que representa el $1.17 \%$. Es importante destacar que el $63.1 \%$ de los jóvenes (135 de los 214) se han infectado mediante el uso compartido de instrumentos de inyección no estériles, o a través de las relaciones sexuales (homo o heterosexuales). Así mismo, tenemos que poner de relieve que entre los infectados por transmisión heterosexual, hay 9 chicas y un sólo varón. Por otra parte, conviene tener en cuenta que los 2.247 jóvenes enfermos de 20 a 24 años (el $12.25 \%$ del total de afectados), probablemente se hayan infectado cuando tenían entre 12 y 16 años, si consideramos los 8 años de latencia que acostumbra a tener el VIH antes de que se comiencen a manifestar los primeros síntomas de la enfermedad (Cf. Sorrell y Springer, 1989).

Por lo que se refiere a Europa, la OMS (1993b) informa que los casos de SIDA comunicados por 35 países hasta finales de marzo de 1993 , fueron de 666 afectados en el grupo de jóvenes de 13 a 19 años (lo que representan el $0.72 \%$ del total de 92.371 enfermos diagnosticados hasta ese momento) y de 6.393 enfermos de 20 a 24 años (el $6.9 \%$ del total).

En los EE.UU., hasta el 31 de diciembre de 1990 (Bowler et al., 1992) los casos de Sida entre adolescentes de 13 a 19 años fueron 629 (472 varones y 157 mujeres), lo que representa el $0.32 \%$ de todos los afectados. El $43 \%$ de las chicas se contagiaron a través de las relaciones heterosexuales (frente a el $1 \%$ de los chicos), mientras que el $35 \%$ de los varones adquirieron la infección por contagio homo o bisexual. Respecto a la prevalencia del Sida entre jóvenes de 20 a 24 años, se contabilizan 6.720 casos (el $3.37 \%$ del total), habiendo adquirido la infección por contacto heterosexual el $30 \%$ de las mujeres y a través de las relaciones homo o bisexuales el $67 \%$ de los varones.

Datos anteriores correspondientes a Nueva York, mostraron que, en esa ciudad, la transmisión heterosexual entre los jóvenes era más frecuente que en los adultos (15\% frente al $8 \%$ ) y que el $46 \%$ de las chicas se habían contagiado de esa forma (Brooks-Gunn et al., 1988; Brooks-Gunn y Furstenberg, 1990).

\section{Otras enfermedades de transmisión sexual (ETS)}

El padecimiento de enfermedades de transmisión sexual (ETS) favorece la infección con el VIH durante las relaciones sexuales sin protección, debido a las llagas y ulceraciones que suelen producir (Cates y Bowen, 1989). Así mismo, 


\section{8}

provocan síntomas más intensos en los sujetos infectados con el VIH (Cameron y Padian, 1990), e incrementan el grado de infecciosidad de éstos sujetos respecto a sus parejas, a causa de la presencia en el semen y las secreciones vaginales de un mayor número de leucocitos portadores del virus, producidos por el estado inflamatorio del tracto genital (Nájera, 1992).

Existen dificultades para conocer la incidencia y prevalencia de las ETS en la mayoría de los países —entre ellos el nuestro- debido a insuficiencias en las reglamentaciones sanitarias para su notificación (Generalitat de Catalunya, 1991). Por este motivo, los datos que podemos ofrecer son más limitados que en el caso del Sida.

En España, en estos últimos años, se ha observado un incremento notable de este tipo de enfermedades, habiéndose detectado que, a lo largo del periodo que va del año 1977 al 1984, la enfermedad pélvica inflamatoria (EPI) — que se produce como consecuencia de la infección con algunas ETS - ha aumentado entre las jóvenes de 14 a 20 años, al menos, el 130.6\%; representando la chlamydia el $60 \%$ de todas las EPI (Olmos, 1989).

Estos datos son explicables teniendo en cuenta que Usieto y Menor (1989) informan de que el $43.6 \%$ de los jóvenes españoles entre 14 y 18 años y el $37 \%$ de los que tienen entre 19 y 21 años no utilizan ningún método de higiene en sus relaciones sexuales, ni siquiera el lavado al terminar el acto sexual.

En Cataluña, el Departament de Sanitat considera preocupante el incremento de la EPI entre las mujeres más jóvenes, especialmente por sus secuelas de embarazos ectópicos, infertilidad y esterilidad (Generalitat de Catalunya, 1991). Otros datos proporcionados por este organismo en la misma publicación, correspondientes a 1701 pacientes que acudieron a un centro sanitario de Barcelona durante el periodo 1984-1989, muestran que aproximadamente el 7\% de los pacientes diagnosticados con alguna ETS son jóvenes entre los 15-19 años, y que el número de mujeres afectadas es casi el doble que el de los varones. Igualmente se ha observado en esta Comunidad Autónoma que los papilomavirus han pasado de afectar a 6.000 personas en 1977 a 30.000 en 1980, siendo este aumento especialmente acusado entre los adolescentes (Olmos, 1989).

También en los EE.UU. las ETS han experimentado un gran crecimiento entre la población general (Cameron y Padian, 1990; Cates y Bowen, 1989). Por lo que se refiere a la población adolescente, según Marwick (1988; Cf. Cochran et al., 1990) dos millones y medio de adolescentes se contagian con alguna ETS cada año en los EE.UU., mientras que Brooks-Gunn et al. (1988) informan de que los mayores porcentajes de mujeres sexualmente activas que sufren enfermedades de transmisión sexual en los EE.UU., tienen edades comprendidas entre los 15 y los 19 años.

\section{Embarazos no deseados}

Como ya mencionábamos en la introducción, los embarazos no deseados es uno de los problemas más graves que afectan a los adolescentes, especialmente a las mujeres, a causa de sus repercusiones tanto individuales como sociales.

Con el incremento de casos de ETS, así como con la aparición del Sida, este problema ha visto incrementada su gravedad por los motivos que expondremos más adelante. Pero antes nos referiremos brevemente a que el índice de embarazos no deseados puede tener, además, un valor informativo respecto a la prevalencia de los comportamientos sexuales de riesgo en la población juvenil: un embarazo no deseado pone de manifiesto que ninguno de los dos miembros de la 


\section{9}

pareja tomó precauciones eficaces para evitarlo, ya fueran píldoras anticonceptivas o preservativos, etc., durante la relación sexual.

En cuanto al agravamiento de las repercusiones de los embarazos no deseados, se ha comprobado que pueden producirse interacciones negativas entre el embarazo y las ETS. En primer lugar, existe el peligro de transmitir la ETS al futuro hijo (en el caso del VIH el contagio se produce en el 30-50\% de los casos) y, por otra parte, el embarazo puede acelerar o agravar las manifestaciones del Sida en la madre (Cameron y Padian, 1990; Cates, 1988; Cates y Bowen, 1989).

Como no disponemos de datos epidemiológicos poblacionales sobre embarazos no deseados — ni sobre abortos legales o ilegales practicados en chicas adolescentes - presentaremos una serie de informaciones que pueden ilustrar la gravedad del problema.

En España, según Olmos (1989), sólo un 20-25\% de los adolescentes españoles de 14 a 20 años, sexualmente activos, usan métodos anticonceptivos. Puerto (1989), en un estudio realizado con 599 mujeres jóvenes que acudieron a diferentes centros de Planificación Familiar de la provincia de Sevilla, encontró que el $45 \%$ de las mujeres que comenzaron sus relaciones sexuales entre los 16-17 años, se quedaron embarazadas a esa misma edad. Por otra parte, la edad más frecuente para el inicio de las relaciones sexuales fue, precisamente, el periodo de 16-17 años (36\%) seguido del de los 18-19 años (30\%).

En fechas más recientes han aparecido en la prensa (Argos, 1993) informaciones provenientes de la Federación de Planificación Familiar de España, basadas en estimaciones estadísticas, según las cuales, siete de cada 100 adolescentes españolas entre los 15 y los 19 años se quedan embarazadas anualmente, y un $8 \%$ de estos embarazos acaban en aborto.

En Cataluña, Pérez (1990) informa que el 10’3\% de las mujeres atendidas en un centro piloto de seguimiento de embarazos en Viladecans (Barcelona) tenían menos de 20 años y que el $40 \%$ de las jóvenes menores de 20 años atendidas en un centro de planificación familiar de Mataró (Barcelona), no utilizaba ningún método anticonceptivo eficaz en sus relaciones sexuales.

En los EE.UU., los embarazos no deseados tienen una elevada incidencia entre los adolescentes. Según Fisher (1990), se calcula que el 30\% de las adolescentes sexualmente activas se quedan embarazadas durante su juventud, y que en el curso de un año se producen 600.000 embarazos no deseados en adolescentes solteras. Boyer y Kegeles (1991) estiman que los embarazos no deseados en adolescentes ascienden al millón, y que las dos terceras partes de las adolescentes de 15 a 19 años sexualmente activas no usan ningún método anticonceptivo. Brooks-Gunn y Furstenberg (1990) comunican que, en una encuesta realizada en 1988, cerca de la tercera parte de los varones adolescentes informó que no había utilizado métodos anticonceptivos en su primera relación sexual. Weisse et al. (1990) exponen que los jóvenes generalmente inician las relaciones sexuales entre los 13 y los 17 años y que menos de la mitad usan anticonceptivos, por lo que el $20 \%$ de las jóvenes entre los 11 y los 19 años quedan embarazadas.

\section{COMPORTAMIENTOS SEXUALES DE RIESGO}

Fundamentalmente se considera como comportamiento sexual de riesgo «las relaciones sexuales con penetración (ya sean vaginales, orales o anales) en las que no se utiliza preservativo». Dado que existe mayor riesgo de rotura de los preservativos en las relaciones anales, este tipo de comportamiento conllevaría mayor 
peligro, sin olvidar que la penetración anal puede ser tanto homo como heterosexual (Nájera, 1992).

Por lo tanto, la mayoría de los programas de prevención del SIDA (Bayés, 1992; Maticka-Tyndale, 1991) recomiendan seguir alguna de las siguientes directrices:

a) Abstinencia sexual absoluta

b) Relaciones sexuales estrictamente monógamas con una pareja fiel no contagiada

c) Prácticas sexuales sin penetración

d) Relaciones sexuales con penetración, pero utilizando siempre preservativo (y espermicidas adecuados) de forma correcta.

En el caso de los adolescentes — y por lo que se refiere al punto b)_ puesto que es poco probable que sus parejas actuales sean ya las definitivas, y debido a que se ha comprobado que interpretan equivocadamente que se puede practicar la monogamia sucesiva (Greig y Raphael, 1989), los consejos preventivos van encaminados a proponer la demora en el inicio de las relaciones sexuales o, alternativamente, a promover el uso del preservativo (Fisher, 1990).

\section{FACTORES INFLUYENTES EN LOS COMPORTAMIENTOS SEXUALES DE RIESGO DE LOS ADOLESCENTES}

Existen diferentes modelos multicausales para explicar los procesos y los factores que intervienen tanto en la preservación de la salud, como en su pérdida (Kirscht, 1983; Rodriguez Marín, 1994). Generalmente, las diferencias que pueden encontrarse entre ellos radican en la importancia preponderante que otorgan a unos u otros factores (biológicos, conductuales, cognitivos, sociales, etc.). En nuestra exposición hemos procurado tener en cuenta los factores considerados relevantes en la mayoría de los modelos.

\section{Factores biológicos}

Nos referiremos en este apartado a determinados cambios fisiológicos que el sujeto produce en su organismo mediante el uso de drogas y que pueden actuar como facilitadores o inhibidores de los comportamientos de riesgo.

\section{Consumo de alcohol}

El consumo de alcohol, antes o durante la relación sexual, favorece los comportamientos sexuales de riesgo, tanto en adolescentes como en adultos (Trocki y Leigh, 1991).

El alcohol ejerce sus efectos de diferentes maneras. Por una parte favorece la interacción social y, por otra, facilita la realización de comportamientos que, en condiciones de abstinencia, el sujeto no se atrevería a llevar a la práctica por temor a sus posibles consecuencias aversivas. Estos efectos son debidos al poder depresor del alcohol sobre el sistema nervioso central, y en concreto sobre los centros encargados de la inhibición de los comportamientos castigados (Swonger y Constantine, 1985). 


\section{1}

FIGURA 1

Relaciones entre diversos influyentes en los comportamientos sexuales preventivos de los adolescentes

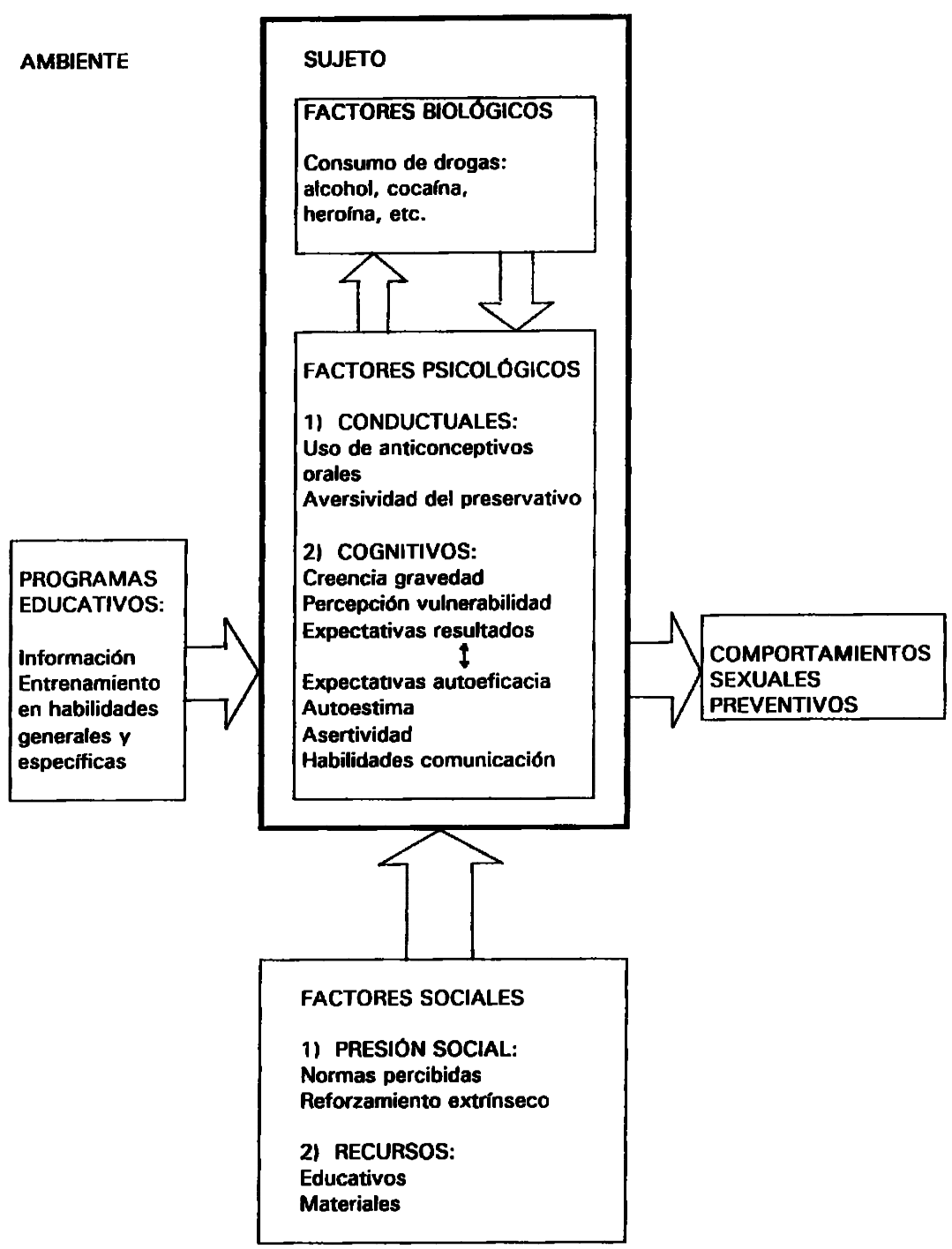

Por otra parte, la creencia de que el alcohol es un afrodisíaco o estimulante sexual - aunque en cierta manera explicable teniendo en cuenta sus efectos iniciales que favorecen la relación social — sería totalmente errónea, puesto que su consumo produce en los hombres dificultades en la erección y eyaculación, mientras que en las mujeres reduce su capacidad orgásmica (Masters et al., 1987). Teniendo en cuenta lo anterior, las adolescentes consumidoras pueden tener mayor riesgo que los varones, puesto que, en su caso, las disfunciones orgánicas causadas por el alcohol no les impedirán mantener relaciones sexuales. 
Algunos estudios realizados muestran que: el alcohol es la droga más ampliamente utilizada por los adolescentes de entre 12 y 17 años (Nathan, 1983); el uso de alcohol por parte de los adolescentes en su primera relación sexual correlacionó con no utilizar métodos anticonceptivos (Cf. Trocki y Leigh, 1991); el uso de alcohol predijo comportamientos sexuales de alto riesgo, en escolares entre los 15 y los 17 años (Keller et al., 1991); el consumo de alcohol aumentó las probabilidades de tener relaciones sexuales, pero disminuyó las probabilidades de utilizar precauciones (Weisse et al. ,1990); y jóvenes partidarios del sexo seguro, realizaron comportamientos de riesgo cuando estaban bajo los efectos del alcohol (Flavin ,1987; Cf. Greig y Raphael, 1989).

\section{Consumo de drogas estimulantes (cocaína, etc.)}

Se considera que la cocaína, por lo general, actúa como un estimulante sexual, aunque también puede tener efectos que alteran y dificultan las relaciones sexuales (priapismo, etc.) (Masters et al., 1987).

La estimulación que produce la cocaína tiene una duración corta (unos 15 minutos) y es frecuente que se repitan las tomas y se procure potenciar sus efectos a través de las inyecciones en vena. Teniendo en cuenta, además, que el precio de la cocaína es elevado, suele producirse el intercambio de sexo por droga, y dadas las propiedades estimulantes de esta sustancia, también se realizan un mayor número de actos sexuales, lo que finalmente se puede traducir en un incremento de los comportamientos sexuales de riesgo. Por ejemplo, se ha comprobado que el uso del crack ha incrementado la difusión del sida heterosexual en los EE.UU.(Cf. Friedman et al., 1990).

\section{Consumo de heroína}

La heroína es una droga depresora del sistema nervioso central, capaz de producir analgesia y euforia (Swonger y Constantine, 1985).

La heroína tiene importantes efectos sobre el comportamiento sexual: en el caso de los varones produce dificultades de erección y de eyaculación, y disminución del interés sexual, mientras que en las mujeres provoca disfunción orgásmica e, igualmente, reducción del interés sexual (Masters et al., 1987).

Aunque los efectos de la heroína, en principio, más bien deberían de reducir los comportamientos sexuales de riesgo, lo que suele ocurrir es que muchos adictos/as se dedican a la prostitución homo/heterosexual como una forma de obtener ingresos con que pagarse la droga (Bravo y De la Fuente; 1991).

Por otra parte, las mujeres adictas no suelen usar anticonceptivos, ya que el consumo de opiáceos provoca amenorrea y disminuye, por esta causa, su motivación para utilizarlos (Cochran y Mays, 1989).

\section{Factores comportamentales}

\section{Anticonceptivos orales}

Se ha comprobado en diferentes estudios que el uso de anticonceptivos orales interfiere con la prevención de las enfermedades de transmisión sexual. Según Fisher (1990), el uso de anticonceptivos por parte de los adolescentes hasta los 
años 80 , ha seguido una pauta regular de desarrollo: al iniciar las relaciones sexuales los adolescentes no usan ningún método anticonceptivo (ni se previenen embarazos ni ETS); después usan preservativos (que son eficaces tanto para prevenir embarazos como ETS); y finalmente usan anticonceptivos orales (que sólo son útiles para evitar embarazos). En opinión del autor, este cambio hacia métodos globalmente menos protectores de la salud se ha debido, principalmente, a que los centros sanitarios prestaban mayor atención a evitar de manera eficaz los embarazos que las ETS. Ford (1992) en una investigación realizada con una muestra de 2955 jóvenes ingleses de 16 a 24 años, encontró que cuando la relación de una pareja se hacía estable y se incrementaba la frecuencia de relaciones sexuales, los jóvenes abandonaban el uso del preservativo en favor de los anticonceptivos orales.

En España también se ha observado este cambio entre las adolescentes atendidas en un centro de asistencia para jóvenes de Mataró. Inicialmente utilizaban el preservativo el $35 \%$ de las 669 jóvenes atendidas, pero como muchas de ellas optaron por cambiar a la píldora, el uso del preservativo quedo reducido al $11 \%$ (Pérez, 1990)

\section{Aversividad del preservativo}

Por lo general, se considera que los preservativos interfieren con el desarrollo de la relación sexual y reducen el placer, aunque lo más frecuente es que las quejas provengan de los varones (Ford, 1992; Weisse et al.,1990). Para compensar estas críticas, algunos autores han puesto de relieve que el uso de preservativos puede tener también efectos positivos, ya que al mantener durante más tiempo la erección, posibilitan que la relación sexual sea más prolongada (Felman, 1979; Cf. Weisse et al., 1990). Oswald y Pforr (1992) comunican que los adolescentes que usan preservativos con regularidad, no consideran que éstos reduzcan el placer, lo que sugiere que su uso continuado posiblemente produce habituación.

\section{Factores cognitivos}

\section{Creencias sobre los riesgos de las relaciones sexuales sin precauciones}

Una de las estrategias empleadas con mayor frecuencia para intentar hacer disminuir la alta incidencia de ETS y de embarazos no deseados, consiste en proporcionar a los jóvenes información sobre los métodos eficaces de prevención. Existe, sin embargo, acuerdo entre los investigadores (Bandura, 1990; Basch, 1989; Bayés, 1992; Brooks-Gunn y Furstenberg, 1990; Diclemente, 1991; Keller et al., 1991; Ross et al., 1989; Weisse et al., 1990) en considerar que la información es una condición necesaria pero no suficiente para iniciar y/o mantener comportamientos preventivos.

Fisher (1990) señala que, por lo general, es bastante frecuente que los adolescentes tengan información poco relevante, errónea o inadecuada sobre la concepción, la anticoncepción y las ETS; por ejemplo, pueden conocer muchos detalles anatómicos y desconocer, sin embargo, cuales son los días fértiles del ciclo menstrual. En una investigación de carácter nacional realizada por Marwick en 1988 (Cf.: Cochran et al. ,1990 y Weisse et al., 1990), el 40\% de los 11.000 adolescentes examinados no conocían los síntomas básicos de una ETS (emisión de fluidos o dolor en los órganos sexuales) y más del $50 \%$ creían que lavarse después del 
coito reducía el riesgo de infección con el HIV e ignoraban que tomar píldoras anticonceptivas no protegía del contagio con una ETS.

Fundamentalmente, los programas existentes se dirigen a incrementar el conocimiento de los adolescentes sobre los comportamientos sexuales de prevención y de riesgo, y a fomentar actitudes preventivas. Estos programas suelen tener éxito en sus objetivos informativos, pero su influencia para cambiar los comportamientos de riesgo es limitada. Por ejemplo, Kegeles et al. ( 1988; Cf. Greig y Raphael, 1989) y Rickert et al. (1989) encontraron que los adolescentes percibían adecuadamente el valor de los condones en la prevención de las ETS y que eran conscientes de la importancia de evitar esas enfermedades, sin embargo, eso no implicaba que se preocuparan de adquirirlos o incrementaran su uso.

Se suele argumentar que estos programas no consiguen modificar los comportamientos sexuales peligrosos, entre otros, por los siguientes motivos: una orientación excesivamente racionalista, poca implicación de los centros educativos en lo que va más allá de la mera información, y falta de acuerdo entre profesores, padres y autoridades políticas y religiosas respecto al contenido de los programas (Bowler et al., 1992; Brooks-Gunn y Furstenberg, 1990).

Respecto a las controversias sobre la moralidad de los programas, Weisse et al. (1990) y Greig y Raphael (1989) opinan que sugerir que todos los adolescentes deberían abstenerse de tener relaciones sexuales, o demorarlas, es una opción poco realista que, en cierta forma, puede provocar reacciones en contra de la prevención si los jóvenes lo interpretan como un discurso moralista o autoritario.

\section{Creencias sobre el propio riesgo (invulnerabilidad)}

Se ha comprobado repetidamente que los jóvenes poseen actitudes y creencias de invulnerabilidad y, por consiguiente, se preocupan muy poco por su salud, lo que contribuye a que coman mal, beban y fumen mucho, conduzcan de forma imprudente, y les gusten las actividades peligrosas (Greig y Raphael, 1989).

Por ejemplo, en un estudio realizado por Weisse et al. (1990) la mitad de los jóvenes comunicó que no se preocupaban por su salud y que, además, no consideraban que fuera responsabilidad suya.

Probablemente esas creencias sean debidas al hecho de que a los adolescentes les falta experiencia de la vida en general. Respecto al papel que juega la experiencia en el aprendizaje de los comportamientos de prevención frente a las enfermedades de transmisión sexual (ETS), Cochran y Peplau (1988; Cf. Cochran y Mays, 1989) observaron que las mujeres que habían modificado su conducta sexual tenían más experiencia sexual que las que no lo habían hecho; siendo el mejor predictor haber recibido previamente tratamiento para una enfermedad de transmisión sexual.

Por lo que se refiere a los embarazos, según Fisher (1990), algunas adolescentes creen que son demasiado jóvenes para quedarse embarazadas, mientras que otras piensan que tener un bebé es siempre algo emocionante, o que puede servir para solucionar todos sus problemas.

\section{Expectativas de resultados y expectativas de autoeficacia}

Según Bandura (1990) resulta más probable que una persona realice una determinada tarea si confía en su capacidad para llevarla a cabo (expectativas de autoeficacia). 
Por lo que se refiere a los comportamientos preventivos, se ha comprobado que los sujetos que tienen unas expectativas de autoeficacia altas y que también confían en la bondad de los resultados del comportamiento de prevención (expectativas de resultados) tienen mayores probabilidades de realizar dichos comportamientos (Weisse et al., 1990).

Según Basch (1989), una forma de adquirir autoconfianza consistiría en ir realizando el aprendizaje a pequeños pasos que faciliten el éxito. Otra forma de incrementar las expectativas de autoeficacia se basaría en el entrenamiento de los sujetos mediante «role playing» y ensayos situacionales, que les permitieran practicar el comportamiento preventivo; habiéndose comprobado que estas estrategias resultaban eficaces para enseñar a negociar el sexo seguro, o a negarse a mantener relaciones sexuales de riesgo (Bandura, 1990). Así mismo, resulta útil emplear modelos que muestren cómo realizar determinadas acciones preventivas y que verbalicen las preocupaciones, dudas o miedos que sienten en esas situaciones que, finalmente, resuelven de manera satisfactoria.

\section{Habilidades de comunicación, asertividad y autoestima}

Teniendo en cuenta que el comportamiento sexual es un comportamiento social, para que sean posibles los comportamientos sexuales de prevención es preciso que los sujetos posean niveles suficientes de autoestima y asertividad, así como estrategias efectivas de comunicación interpersonal con la pareja (Welch $e t$ al., 1990). Aunque las habilidades para la comunicación tienen una importante base cognitiva, también podrían ser clasificadas como un recurso que depende de la educación.

Fisher (1990) propone como una de las etapas del comportamiento preventivo, la discusión y negociación con la pareja sobre los métodos que se van a utilizar para prevenir los embarazos o las ETS, procurando conseguir el acuerdo e interés de la pareja en la prevención o, en caso contrario, que el sujeto se niegue a llevar a cabo comportamientos de riesgo.

Weisse et al. , (1990) exponen que algunos adolescentes no se atreven a pedir a su pareja que utilice el preservativo, o les falta la asertividad necesaria para conseguir que su uso sea regular y efectivo.

Entre las diversas sugerencias preventivas, ha sido frecuente que se aconsejara a los sujetos que se interesaran por conocer la historia de riesgo de su pareja, lo que requiere también de una serie de habilidades de comunicación.

Diclemente (1991) en un estudio transversal realizado con 112 adolescentes encarcelados menores de 19 años, encontró que los jóvenes que hablaron con sus parejas sobre el Sida antes de mantener relaciones sexuales, era más probable que hubieran utilizado preservativo que quienes no hablaron.

Sin embargo, algunos estudios realizados por Cochran y Mays (1990) y Welch et al., (1990) alertan sobre la posibilidad de que los sujetos puedan mentir con tal de mantener relaciones sexuales sin problemas, o bien, utilicen la conversación sobre temas relacionados con el Sida para intentar ganarse la confianza de la pareja.

En cuanto a la autoestima, se ha observado que las personas que sienten poco respeto hacia sí mismas y que experimentan falta de control hacia sus propias vidas, no suelen responder a los mensajes para mejorar la salud (Greig y Raphael, 1989). Así mismo, es probable que las personas que se sienten perseguidas y rechazadas por la sociedad no cooperen en los esfuerzos preventivos. Por contra, Vicent et al. (1987; Cf. Greig y Raphael, 1989) obtuvieron éxito en la preven- 
ción de embarazos entre las adolescentes con un programa comunitario que potenciaba la autoestima y la responsabilidad personal.

\section{Factores psicosociales}

\section{Presión social}

Anteriormente nos hemos referido a la naturaleza social del comportamiento sexual, poniendo énfasis en los factores personales protectores. En este apartado trataremos de los diferentes tipos de influencia social que se suele ejercer sobre dicho comportamiento.

En opinión de Ross et al. (1989) y de Bandura (1990), las conductas recomendadas para reducir la transmisión del VIH (uso del preservativo, alteración de comportamientos sexuales o de su frecuencia), están sujetas a las presiones y normas de los compañeros, por lo que pueden existir dificultades para que el sujeto que desea hacer prevención sea asertivo y mantenga su conducta. Según Basch (1989), mantener relaciones sexuales sin protección podría ofrecer a los jóvenes poderosos reforzadores: aprobación social — real o imaginada-, diversión, excitación, placer, una forma de expresar rebeldía o independencia, de demostrar valentía, virilidad o machismo, de afrontar emociones, etc., mientras que hacer prevención sólo les supondría evitar enfermedades exponiéndose, por otra parte, a ser rechazado por los compañeros.

Por ejemplo, Diclemente (1991) en el estudio anteriormente mencionado, comprobó que los jóvenes que creían que la mayoría de sus compañeros utilizaban el preservativo (normas de los pares percibidas) empleaban esta precaución en mayor proporción que quienes creían que era utilizado por pocos.

Como las presiones del grupo pueden ser especialmente fuertes en el caso de los adolescentes, Bandura (1990), Cates y Bowen (1989) y Basch (1990) opinan que los programas preventivos deberían entrenar a los adolescentes para que sean capaces de negociar con éxito el uso de precauciones, y plantean que se podría proporcionar reforzamiento positivo para mantener los comportamientos preventivos. Basch (1990) sugiere la creación de grupos de «ayuda mutua» (similares a los que ya existen para otros problemas de salud).

Weisse et al. (1990) y Cates y Bowen (1989) proponen que, simultáneamente a estas intervenciones, se intenten cambiar las normas del grupo mediante campañas que, poco a poco, modifiquen las percepciones negativas sobre la prevención que actualmente prevalecen (de manera similar a lo que ha ocurrido con el consumo de cigarrillos).

En algunas intervenciones para potenciar el uso de anticonceptivos entre las adolescentes sexualmente activas, se ha observado que la existencia una buena comunicación de la adolescente con sus padres y un cierto grado de permisividad por parte de éstos, influía en el uso regular de los anticonceptivos; otro factor predictivo fue el apoyo a la prevención por parte de la pareja de la adolescente (Kasner, 1984; Cf.: Weisse et al., 1990).

\section{Insuficiencia de recursos}

Consideramos recursos necesarios para la prevención tanto los que dependen de las instituciones sociales (programas de sensibilización, educación, etc.), como las habilidades y los materiales que el sujeto precisa para llevarla a cabo. 
En general, los recursos educativos y sanitarios, todavía son insuficientes para proporcionar a los adolescentes los conocimientos, entrenamiento y materiales básicos necesarios para realizar los comportamientos sexuales de prevención. Además, la mayoría de las intervenciones se han debido al interés y al voluntarismo de profesores, médicos, psicólogos, asistentes sociales y otros profesionales.

Respecto a los adolescentes que corren mayor riesgo (adictos a las drogas por vía parenteras y sexualmente activos) creemos que necesitan urgentemente programas preventivos específicos (Cf. Planes, 1991, 1992).

\section{CONCLUSIONES}

En la revisión epidemiológica hemos podido comprobar que España tiene unos porcentajes de adolescentes enfermos de Sida (1.17\%) superiores a la media europea $(0.72 \%)$, que las autoridades sanitarias juzgan preocupantes los índices de ETS observados en las mujeres más jóvenes y que muchas adolescentes se quedan embarazadas porque no utilizan ningún método anticonceptivo.

Estos datos sugieren que sería conveniente unificar los programas para la prevención simultánea del SIDA, las otras ETS y los embarazos no deseados, maximizando de esta forma los recursos disponibles. Dichos programas, además de tener en cuenta los aspectos informativos, deberían posibilitar la adquisición de habilidades preventivas y proporcionar los recursos necesarios.

En su diseño sería conveniente contemplar la influencia de los factores biopsico-sociales anteriormente revisados. De entre ellos destacaríamos tres:

1) Las habilidades de comunicación: Se debería enseñar a los adolescentes a negociar con eficacia ya sea la demora de las relaciones sexuales, o el uso del preservativo.

2) La habituación al preservativo: Se debería conseguir que los adolescentes sexualmente activos que han comenzado a utilizar el preservativo, mantengan su uso el tiempo necesario hasta que dejen de percibir las molestias que inicialmente les haya podido causar.

3) Presión social: Habría que lograr que tanto la pareja, como los amigos y compañeros del adolescente aceptaran positivamente la prevención, reforzando explícitamente los comportamientos preventivos del sujeto si fuera preciso.

En cuanto a la aplicación de los programas, creemos que se debe hacer un esfuerzo por acercarlos al máximo a sus destinatarios, a través de los centros educativos, sanitarios, lugares de diversión, etc., puesto que, por lo general, los adolescentes no son conscientes del peligro que corren, o sienten vergüenza o temor a exponer sus preocupaciones o, sencillamente, ignoran cómo buscar y conseguir ayuda. Tampoco hay que olvidar que son legalmente menores de edad y están bajo tutela.

Por último, creemos que los resultados de los programas se deberían evaluar, fundamentalmente, a través de los cambios conductuales que hayan producido, ya sea utilizando los autoinformes de los propios adolescentes, o mediante el conocimiento de la incidencia de embarazos o de ETS entre los adolescentes que hayan seguido el programa. 


\section{8}

\section{Referencias}

Argos, L. (1993, julio 7). Siete de cada 100 adolescentes españolas se quedan embarazadas cada año. El País, p. 31.

BandurA, A. (1990). Perceived self-efficacy in the exercise of control over AIDS infection. Medienpsychologie, 1, 23-43.

BASCH, Ch. E. (1989). Preventing AIDS through education : Concepts, strategie, and research priorities. Journal of School Health, 59 (7), 296-300.

BAYES, R. (1992). Aportaciones del análisis funcional de la conducta al problema del SIDA. Revista Latinoamericana de Psicología, 24 (1-2), 35-56.

BLAKE, D. (1991). The HIV/AIDS situation in the world and the Global Programme on AIDS. Publicación Oficial de la Sociedad Española Interdiscipli naria de SIDA, 2(5), 74-75.

Bowler,S.; SheON, A. M.; D'ANGelo, L. J., y Vermund, S. H. (1992). HIV and AIDS among adolescents in the Unitet States: Increasing risk in the 1990s. Journal of Adolescence, 15 (4), 345-371.

Boyer, CH. B., y KEGELES, S. M. (1991). AIDS risk and prevention among adolescents. Society of Scientific Medicine, 33 (1), 11-23.

Bravo, M. L., y De LA Fuente, L. (1991). Epidemiología de la infección por VIH en los usuarios de drogas por via parenteral. Publicación Oficial de la Sociedad Española Interdisciplinaria de SIDA, 2(8), 335-342.

Brooks-Gunn, J.; Boyer, CH. B., y Hein, K. (1988). Preventing HIV infection and AIDS in children and adolescents. American Psychologist, 43 (11), 958-964.

Brooks-Gunn, J., y Furstenberg, F. F. (1990). Coming of age in the era of AIDS: Puberty, sexuality, and contracepcio n. The Milkbank Quarterly, 68 (supl. 1), 59-84.

Caceres, C. F.; Rosasco, A. M.; Muñoz, S.; Gotuzzo, E.; Mandel, J., y Hearst, N.(1992). Necesidades educativas en relación con la sexualidad humana y el SIDA entre estudiantes y profesores de Escuela Secundaria en Lima. Revista Latinoamerican a de Psicología, 24 (1-2), 109-122.

Cameron, W., y Padian, N. S. (1990). Sexual transmission of HIV and the epidemiology of other transmitted diseases. AIDS, 4, S99-S103.

CATES, W. (1988).The "other STD's": do they really matter?. JAMA, 259 (24), 3606-3608.

CATES, W., y Bowen, S. G. (1989). Education for AIDS prevention: not our only voluntary weapon. American Journal of Public Health, 79 (7), 871-874.

Cochran, S. D., y MAYs, V. M. (1989). Women and AIDS-related concerns. American Psychologist, 44(3), 529-535.

Cochran, S. D., y Mays, V. M. (1990). Sex, lies, and VIH. The New England Journal of Medicine, 322 (11), 774-775.

Cochran, S. D.; Keidan, J., y Kalechstein, A. (1990). Sexually transmitted diseases and acquired immunodeficien cy syndrome (AIDS). Sexually Transmitted Diseases, 17 (2), 80-86.

Diclemente, R. J. (1991). Predictors of HIV-preventive sexual behavior in a high-risk adolescent population: The influence of perceived peer norms and sexual communication on incarcerated adolescents' consistent use of condoms. Journal of Adolescent Health, 12 (5), 385-390.

FISHER, J. D. (1990). Understanding and preventing adolescent pregnancy and sexually transmissible disease. En J. Edwards; R.S. Tindale; L. Heath y E.J. Posavac (Eds.). Social influence processes and prevention (pp. 71-101). Nueva York: Plenum Publishing Corporation.

FORD, N. (1992). The AIDS awareness and sexual behaviour of young people in the South-west of England. Journal of Adolescence, 15 (4), 393-413.

Friedman, S. R.; Des Jarlais, D. C.; Sterk, C. E.; Sotheran, J. L.; Tross, S.; Woods, J.; Sufian, M., y ABDUL-QUADER, A. (1990). AIDS and the social relations of intravenous drug users. The Milkbank Quarterly, 68, 85-110.

Generalitat de Catalunya (1991). Guia per a la prevenció i control de les malalties de transmissió sexual. Barcelona: Departament de Sanitat i Seguretat Social.

Greig, R., y RAPHAEL, B. (1989). AIDS prevention and adolescents. Community Health Studies, 13 (2), 211-219.

Keller, S. E.; Barlett, J. A.; Schleifer, S. J.; Johnson, R. L.; Pinner, E., y Delaney, B. (1991). HIV-relevant sexual behavior among a healthy inner-city heterosexual adolescent population in an endemic area of HIV. Journal of Adolescent Healh, 12 (1), 44-48.

KIRSCHT, J. P. (1983). Preventive health behavior: A review of research and issues. Health Psychology, 2 (3), 277-301.

Masters, W. H.; Johnson, V. E., y Kolodny, R. C. (1987). La sexualidad humana 3. Perspectivas clínicas y sociales. Barcelona: Grijalbo.

MatickA-Tyndale, E. (1991). Modification of sexual activities in the era of AIDS: A trend analysis of adolescent sexual activities. (Accepted for publication in Youth and Society, september, 1991).

Mendoza, R. (1984). Un renacimiento esperanzador. Cuadernos de Pedagogía. Monográfico Educación para la Salud,(115-116), 4-7.

Najera, R. (1992). Epidemiología. En R. Nájera (Ed.), Sida. De la biomedicina a la sociedad. (pp.115163). Madrid: EUDEMA.

Nathan, P. E. (1983). Failures in prevention. American Psychologist, April, 459-467.

OLmOS, L. (1989). Las enfermedades de transmisión sexual en los adolescentes, Jano, 37 (879), 77-80. 
O.M.S. (1993a). Información Epidemiológica. Publicación Oficial de la Sociedad Española Interdiscipl inaria de SIDA, 4 (7), 432-441.

O.M.S. (1993b). Información Epidemiológica. Publicación Oficial de la Sociedad Española Interdisciplinaria de SIDA, 4 (3), 481-490.

Oswald, H., y PFOrR, P. (1992). Sexuality and AIDS: attitudes and behaviors of adolescents in East and West Berlin. Journal of Adolescence, 15 (4), 373-391.

Perez, M. (1990, enero 21). "Pasando" de anticoncept ivos. El País, p.22.

Planes, M. (1991). La magnitud y la demora en las consecuencias como variables críticas en el autocontrol: Aplicación al caso de comportamientos de riesgo al VIH. Tesis doctoral microfilmada. Universidad Autónoma de Barcelona, Bellaterra.

Planes, M. (1992). Influencia del conocimiento de los resultados de la prueba de anticuerpos frente al virus de inmunodefici encia humana (VIH), en los comportamientos de prevención de los adictos a las drogas por via parenteral (ADVP). Revista de Psicología de la Salud, 4 (2), 103-114.

Puerto, L. (1989). Planificación familiar: Un estudio en jóvenes sevillanos. Apuntes de Psicología, (2829), 25-28.

Rickert, V. I.; JAY, M. S.; Gottlleb, A., y Bridges, CH. (1989). Adolescents and AIDS. Female's attitudes and behaviors toward condom purchase and use. Journal of Adolescent Health Care, 10 (4), 313 316.

RoDRIGUEz MARIN, J. (1994). Evaluación en prevención y promoción de la salud. En R. Fernández Ballesteros (Ed.), Evaluación conductual boy. Un enfoque para el cambio en psicología clínica y de la salud (pp. 652-712). Madrid: Pirámide.

Ross, M. W.; CAUdLE, CH., y TAYLOR, J. (1989). A preliminary study of social issues in AIDS prevention among adolescents. Journal of School Health, 59 (7), 308-311.

SORRELl, S. J., y Springer, E. D. (1989). The argument against HIV-antibody testing in chemical dependence treatment programs. Journal of Psychoactive Drugs, 21, 419-421.

Swonger, A. K., y Constantine, L. L. (1985). Drogas y terapia. Madrid:Alhambra.

Trocki, K. F., y LEIGH, B. C. (1991). Alcohol consumption and unsafe sex : A comparison of heterosexuals and homosexual men. Journal of Acquired Immune Deficiency Syndrome, 4 (10), 981-986.

Usieto, R., y MENOR, J. (1989). La adolescencia española y las prácticas de riesgo frente al SIDA. Jano, 37 (879), 91-94.

Welch, R. J.; Freeman, K. E., y Johnson, S. J. (1990). Talk among sexual partners about AIDS. Factors differentiat ing those who talk from those who no dot. Communication research, 17 (6), 792-808.

Weisse, C. S.; Nesselhof-Kendall, S. E.; Fleck-Kandath, C., y Baum, A. (1990).Psychosocial aspects of AIDS prevention among heterosexual s. En J. Edwards; R.S. Tindale; L. Heath y E.J. Posavac (Eds.). Social influence processes and prevention (pp. 39-70). Nueva York: Plenum Publishing Corporation.

\section{Extended Summary}

Adolescents are, today, one of the groups which due to their behavioural, cognitive and social characeristics, run the greatest risk of possible HIV contagion. This threat to the health and well-being of adolescents is added to other problems already facing this group: unwanted pregnancies, sexually transmitted diseases (STD), which also derive from the lack of prevention in sexual relations.

The article presents different biological, psychological (behavioural and cognitive), and social factors that can facilitate, interfere with or impede preventive social behaviour in adolescents. It also reviews and evaluates some preventive intervention programmes that have been carried out.

Among biological factors, drug use (both legal and illegal, stimulants and depressors) negatively influences preventive sexual behaviours. With respect to Psycho-behavioural factors, it seems that an increase in the use of oral contraceptives by adolescent females is in detriment to the use of other methods of contraception, such as condoms. This means that the triple effectiveness of the latter (in terms of preventing pregnancies, STD, and AIDS), benefitial to both members of the couple, is wasted. In addition, male adolescents are frequetly hostile to using contraceptives as they reduce pleasurable sensations. In terms of $P$ sychocognitive factors, information is a necessary but insufficient factor for initiating and/or mantaining preventive behaviours which are also influenced by other fac- 
tors, such as: risk perception, belief in one's own invulnerability, and expectations both towards results and effectiveness of the preventive method adopted (together with their ability of putting them into practice correctly). Other factors that also negatively affect preventive sexual behaviours are: low self-esteem, poor assertiveness and communication abilities. Finally, in relation to social factors, peer-pressure — both in favour and against prevention- exerts a decisive influence on whether an adolescent decides to adopt preventive methods or not. Likewise, belonging to economically and culturally disfavoured social groups makes prevention more difficult due to poor material (price of male contraceptives) and educational resources.

From this review, the discussion supports the advisability of unifying current intervention programmes so that it may be possible to simultaneously prevent sexual risk behaviours and their corollaries, thus maximizing available resources. Such programmes should take into account the following aspects:

- The need to approach adolescents through education centres, youth clubs, etc., for in general they are unaware of the dangers they are exposed to, they may also be afraid or embarrassed to talk about their worries, and/or they may simply not know where and how they may get help.

- Intervention programmes should not be restricted to distributing information, they should also facilitate the acquisition of preventive behaviours and make resourses available.

- Intervention programmes should cover all possible levels of prevention: primary, secondary, and tertiary.

- Finally, the success of intervention programmes should be evaluated through assessment of behavioural changes that have taken place; either through the use of adolescents' self-reports, or through the knowledge of the incidence of pregnancies and STD among adolescents in the programme. 